\title{
Major Degradation Product Identified in Several Pharmaceutical Formulations against the Common Cold
}

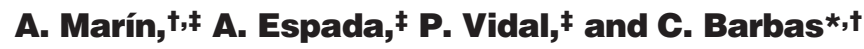 \\ Facultad de CC Experimentales y de la Salud, Universidad San Pablo-CEU, Urbanización Montepríncipe, \\ Boadilla del Monte, 28668 Madrid, Spain, and European Analytical Technologies, DCRT Alcobendas, Lilly S.A., \\ Avda. de la Industria 30, Alcobendas, 28108 Madrid, Spain
}

Different pharmaceutical preparations against the common cold containing acetaminophen, phenylephrine hydrochloride, and chlorpheniramine have been studied. An unidentified degradation product has been discovered in these preparations after short- and long-term stability studies, with increasing concentration at increasing storage temperatures and times. LC/MS was employed to identify and obtain molecular weights of the degradation products using an electrospray ionization interface. To obtain detailed structural information on the major degradation product, its isolation by SPE followed by HPLC was required. Final isolation was accomplished by using a new pentafluorophenyl stationary phase. Once the major degradation product was isolated, MS/MS, ${ }^{1} \mathrm{H}$ NMR, ${ }^{13} \mathrm{C}$ NMR, COSY, HMBC, and HSQC techniques permitted the complete characterization of this new compound formed under the usual stability conditions at climatic chambers. Its origin was also established as a result of the interaction between two of the components in the formulation. It is important to remark that this compound has not been described in the literature up to now and that, moreover, it has been identified in several commercial preparations containing phenylephrine hydrochloride and chlorpheniramine maleate.

Stability assays of drug products are performed to ensure the absence of degradation products at a determined level. Pharmaceutical formulations against the common cold usually contain acetaminophen, phenylephrine hydrochloride, and chlorpheniramine maleate. In previous works carried out at our laboratory to study these pharmaceutical formulations, new analytical methods were developed using HPLC and CE. ${ }^{1-4}$ Moreover, impurities and degradation products of acetaminophen, phenylephrine hydrochloride, and chlorpheniramine maleate have long been studied. ${ }^{5-14}$ Despite this large number of works, nothing has been

\footnotetext{
* Corresponding author. Fax: 003491 3510475. E-mail: cbarbas@ceu.es.

$\dagger$ Universidad San Pablo-CEU.

* Lilly S.A.

(1) Marín, A.; García, E.; García, A.; Barbas, C. J. Pharm. Biomed. Anal. 2002, 29, 701-714.

(2) Olmo, B.; García, A.; Marín, A.; Barbas, C. J. Chromatogr., A, In press.

(3) Marín, A.; Barbas, C. J. Pharm. Biomed. Anal. 2004, 35, 769-777.

(4) García, A.; Rupérez, F. J.; Marín, A.; de la Maza, A.; Barbas, C. J. Chromatogr., $B$ 2003, 785, 237-243.
}

reported on the interactions among the active compounds aforementioned when contained together in a pharmaceutical formulation.

Interestingly, when developing a short-term stability assay with these specialities at our laboratory, an unidentified degradation product, which did not appear during forced degradation assays, appeared with increasing concentration at increasing storage temperature and time in some formulations. It is well known that the study and characterization of degradation products from drugs is of paramount importance, since they can not only reduce the effectiveness of the formulation but, in some cases, these degradation products can be harmful for human health. The United States Food and Drug Administration (FDA) and other regulatory agencies around the world require that impurities in drug substance and drug product at levels recommended by the International Conference on Harmonization (ICH) must be isolated and characterized. Identifying process-related impurities and degradation products also helps us to understand the production of impurities and assists in defining degradation mechanisms.

Therefore, the objective of the present work was the identification, isolation, and characterization for the first time of an unknown degradation product formed in diverse pharmaceutical formulations against the common cold containing as active compounds acetaminophen, phenylephrine hydrochloride, and chlorpheniramine maleate. To do this, a completely new analytical protocol is developed. Namely, different analytical tools were employed including isolation with solid-phase extraction (SPE), separation by HPLC and CE, and solute characterization by spectroscopic techniques coupled on-line with HPLC, e.g., LC/ MS, or directly used off-line (MS/MS, ${ }^{1} \mathrm{H}$ NMR, ${ }^{13} \mathrm{C}$ NMR, COSY, HSQC, HMBC).

\footnotetext{
(5) Troup, A. E.; Mitchner, H. J. Pharm. Sci. 1964, 53, 375-379.

(6) Marín, A.; Barbas, C. J. Pharm. Biomed. Anal. 2004, 35, 1035-1045.

(7) Sune, J. M.; Jurado, F.; Lizcano, J.; Salvado, M. A.; García, M. J.; Miro, M. L. Cienc. Pharm. 1992, 2, 401-410.

(8) Fairbrother, J. E. Anal. Profiles. Drug Subst. 1974, 3, 1-109.

(9) Schieffer, G. W.; Hughes, D. E. J. Pharm. Sci. 1983, 72, 55-59.

(10) Gaglia, C. A., Jr. Anal. Profiles Drug Subst. 1974, 3, 483-512.

(11) Millard, B. J.; Priaulx, D. J.; Shotton, E. J. Pharm. Pharmacol. 1973, 25, $24-31$.

(12) Yamato, S.; Nakajima, M.; Kawakami, N.; Shimada, K.; Tachikawa, E.; Ohta, S.; Zenda, H. Chem. Pharm. Bull. 2000, 48, 1205-1207.

(13) Ahmad, M.; Akhtar, N.; Shah, P. A.; Anjum, S. Acta Pharm. Turcica 1999 $41,66-70$.

(14) Eckhart, C. G.; McCorkle, T. Anal. Profiles Drug Subst. 1978, 7, 43-80.
} 


\section{EXPERIMENTAL SECTION}

Chemicals. Standards of actives (acetaminophen, phenylephrine, chlorpheniramine maleate) and impurities (4-aminophenol, 4-chloracetanilide, 4-nitrophenol) as well as capsules and excipients (talk, magnesium estearate, avicell) of the specialities were kindly provided by CINFA, S.A. (Pamplona, Spain). Sodium hydroxide was from Panreac (Barcelona, Spain), dimethyl sulfoxide (DMSO) was from Scharlab (Barcelona, Spain), phosphoric acid, ammonium acetate, acetonitrile (HPLC), and sodium dodecyl sulfate (SDS) were from Merck (Darmstadt, Germany), cetyltrimethylammonium bromide (CTAB) was from Fluka (Madrid, Spain), and water was purified with a Milli-Q plus system from Millipore (Bedford, MA).

Stability Study. The stability study was made with capsules stored in climatic chambers under the following conditions of temperature and relative humidity $(\mathrm{RH})$ : chamber $\mathrm{A}, 25^{\circ} \mathrm{C}$ and $60 \% \mathrm{RH}$; chamber $\mathrm{B}, 30{ }^{\circ} \mathrm{C}$ and $60 \% \mathrm{RH}$; chamber $\mathrm{C}, 40^{\circ} \mathrm{C}$ and $75 \%$ RH. Samples were analyzed at 0,3 , and 6 months.

Standard Solutions and Sample Preparation for Quantization. For HPLC and CE analysis, the solvent solution (SS) for standards and samples was phosphate buffer $20 \mathrm{mM}$ at pH 7.0/ acetonitrile 70:30 (v/v) and for LC/MS was water/acetonitrile 70: $30(\mathrm{v} / \mathrm{v})$ in order to eliminate the problems brought about by the phosphate buffer in the electrospray ionization (ESI)-MS detector. ${ }^{15}$

A stock solution of phenylephrine was prepared with $250 \mathrm{mg}$ of phenylephrine hydrochloride exactly weighed and dissolved with SS in a $25-\mathrm{mL}$ volumetric flask. For chlorpheniramine maleate stock solution, $100 \mathrm{mg}$ was made up to $25 \mathrm{~mL}$ with SS. For the reference stock standard, $500 \mathrm{mg}$ of acetaminophen was weighed into a 50-mL volumetric flask and 1-mL aliquots of phenylephrine and chlorpheniramine solutions were added. The mixture was made up to the corresponding volume with SS and treated with magnetic stirring for $10 \mathrm{~min}$.

For capsules, $610 \mathrm{mg}$ of sample (the approximate weight of one capsule, containing $500 \mathrm{mg}$ of acetaminophen, $10 \mathrm{mg}$ of phenylephrine hydrochloride, $4 \mathrm{mg}$ of chlorpheniramine maleate, and $96 \mathrm{mg}$ of excipients) coming from 20 capsules was dissolved in a 50-mL volumetric flask with SS. After $10 \mathrm{~min}$ of magnetic stirring, an aliquot was filtered with a $0.45-\mu \mathrm{m}$ syringe filtration disk to the vials for injection in the HPLC system. In all cases, three replicates were processed.

HPLC Analysis. The system consisted of an Agilent Technologies 1100 liquid chromatograph (Agilent Technologies, Waldbronn, Germany) with a quaternary pump, an automatic injector, a diode array detector (DAD), and a column oven. The chromatographic method, previously described and validated, ${ }^{4}$ employed a $5-\mu \mathrm{m}$ particle, Discovery HS poly (ethylene glycol) (PEG) column (Supelco, Tres Cantos, Madrid, Spain), $15 \times 0.46 \mathrm{~cm}$, kept at 35 ${ }^{\circ} \mathrm{C}$.

The mobile phase was phosphate buffer $20 \mathrm{mM}$ at $\mathrm{pH} 7.0 /$ acetonitrile 90:10 (v/v). The phosphate buffer was prepared from $\mathrm{H}_{3} \mathrm{PO}_{4}$ by adding $\mathrm{NaOH}$ to reach $\mathrm{pH} 7.0$. The flow rate was $1 \mathrm{~mL} /$ $\mathrm{min}$, and the injection volume was $5 \mu \mathrm{L}$. UV detection was performed at 215 and $254 \mathrm{~nm}$.

(15) Ross, P. Technical bulletin. ThermoHypersil. Keystone Scientific operations. TB 00-11, 1-6.
CE Analysis. Capillary electrophoresis was carried out in a P/ACE 5500 instrument (from Beckman) with DAD. The separation was carried out using an uncoated fused-silica capillary (57 $\mathrm{cm} \times 75 \mu \mathrm{m}$ i.d.). During the development of the analytical method, both polarity modes were tested and parameters such as $\mathrm{pH}$ and concentration of the buffer and the presence and concentration of surfactants were also studied. Two different methods were optimized:

Method 1 employed normal polarity, injection at the positive end (anode), and detection at the negative end (cathode) with $100 \mu \mathrm{A}$ of applied current. Separation buffer was prepared with $40 \mathrm{mM} \mathrm{H}_{3} \mathrm{PO}_{4}$ made up to $\mathrm{pH} 6.20$ with $\mathrm{NaOH}$ and with $0.5 \mathrm{mM}$ SDS added.

Method 2 employed reversed polarity, injection at the negative end (cathode), and detection at the positive end (anode) with 100 $\mu \mathrm{A}$ of applied current. Separation buffer was prepared with $40 \mathrm{mM}$ $\mathrm{H}_{3} \mathrm{PO}_{4}$ made up to $\mathrm{pH} 6.40$ with $\mathrm{NaOH}$ and with $0.5 \mathrm{mM} \mathrm{CTAB}$ added.

In both cases, the injection was by pressure $\left(3.45 \times 10^{-2}\right.$ bar $)$ during $5 \mathrm{~s}$. UV detection was performed at $200 \mathrm{~nm}$. Temperature was maintained at $25^{\circ} \mathrm{C}$. The capillary was flushed between runs with the BGE for 1 min. Fresh new vial buffers were replaced after each six runs.

LC/MS Analysis. HPLC experiments were carried out on an Agilent Technologies 1100 LC. Electrospray mass spectrometry measurements were performed on a MSD quadrupole mass spectrometer (Agilent Technologies, Palo Alto, CA) interface to the 1100 HPLC system. MS measurements were acquired simultaneously in both positive and negative ion full-scan modes, and detailed conditions were previously described. ${ }^{6}$ Briefly, the chromatographic analysis was performed on a Discovery HS PEG column $150 \times 4.6 \mathrm{~mm}, 5 \mu \mathrm{m}$ (Supelco), kept at $35^{\circ} \mathrm{C}$. The mobile phase was $10 \mathrm{mM}$ ammonium acetate at $\mathrm{pH}$ 7/acetonitrile 90:10 $(\mathrm{v} / \mathrm{v})$. UV detection was performed at 215 and $254 \mathrm{~nm}$, and the injection volume was $5 \mu \mathrm{L}$.

SPE Isolation. For the isolation by SPE, Oasis HLB ( $6 \mathrm{~g})$ cartridges from Waters were used. The mobile phase was $10 \mathrm{mM}$ ammonium acetate at $\mathrm{pH} 7$ (A) and methanol (B). A 4.5-g aliquot of sample was solved in $5 \mathrm{~mL}$ of a mixture $\mathrm{H}_{2} \mathrm{O} / \mathrm{DMSO}$ 1:1 (v/v). The mixture was centrifuged at $4000 \mathrm{rpm}$ for $5 \mathrm{~min}$, and the supernatant was distributed in eight cartridges, loading $500 \mu \mathrm{L}$ in each one. Cartridges were eluted in parallel with a nine -tep gradient from 100 to $95 \% \mathrm{~A}$. The collected fractions were concentrated in a Genevac evaporator until dryness.

HPLC Isolation. For the isolation of the degradation product by HPLC, the following chromatographic method was employed: The column was an HS F5 $100 \times 10 \mathrm{~mm}, 5 \mu \mathrm{m}$ (Supelco), kept at $35{ }^{\circ} \mathrm{C}$. The mobile phase was $10 \mathrm{mM}$ ammonium acetate at $\mathrm{pH}$ 7/acetonitrile 40:60 (v/v). The flow rate was $5 \mathrm{~mL} / \mathrm{min}$, and the injection volume was $50 \mu \mathrm{L}$. UV detection was performed at 215 .

MS/MS. MS/MS experiments were carried out on an ion trap mass spectrometer (Agilent Technologies, Palo Alto, CA) with the isolated and purified compound. The polarity was positive. Drying gas flow and temperature were $5 \mathrm{~L} \cdot \mathrm{min}^{-1}$ and $300{ }^{\circ} \mathrm{C}$, respectively. The nebulizador pressure was $11 \mathrm{psi}$ and the current corona 4000 nA.

NMR. NMR experiments were acquired on a Bruker Avance 500 spectrometer in $\mathrm{D}_{2} \mathrm{O}$ at 298 and $333 \mathrm{~K}$ with a broadband 


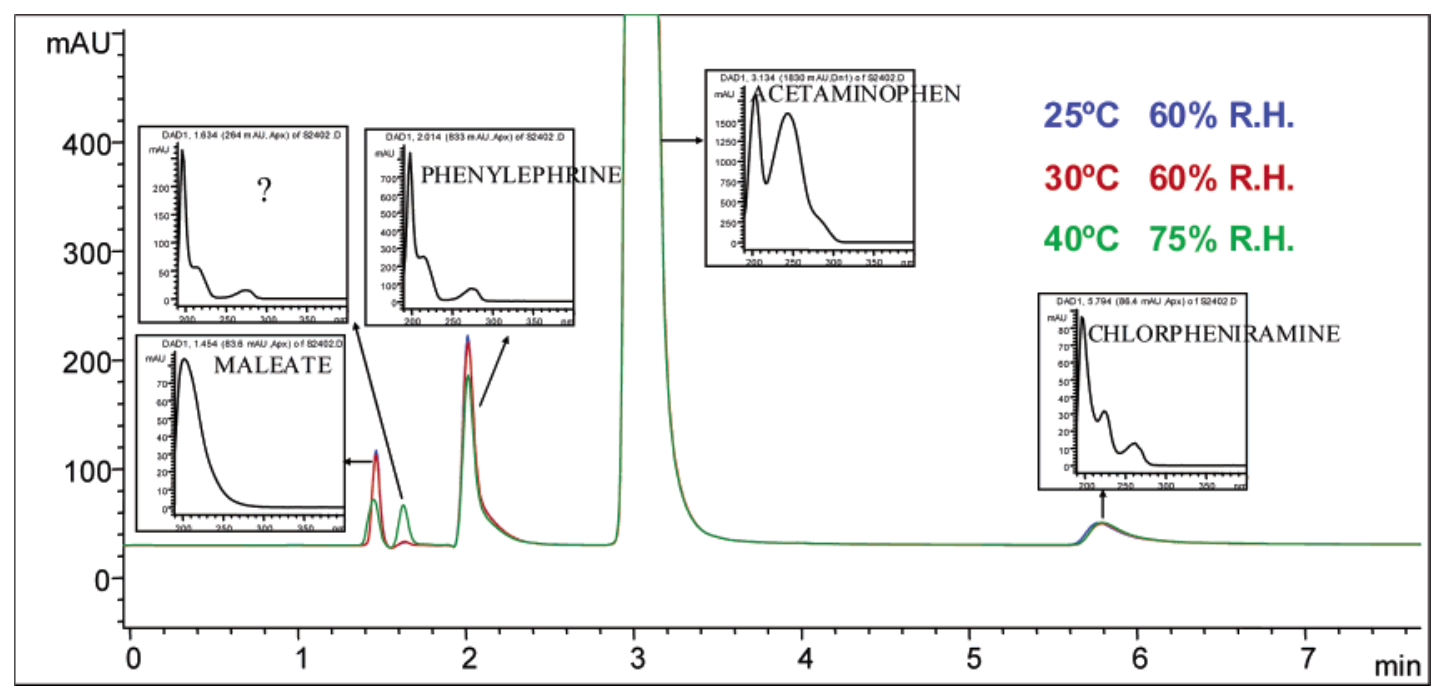

Figure 1. Chromatograms of capsules after 6 months stored in climatic chambers at different temperatures and RH. HPLC-UV method: Discovery HS PEG column $(5 \mu \mathrm{m}, 4.6 \times 150 \mathrm{~mm})$. Mobile phase: $90 \%(20 \mathrm{mM}$ phosphate buffer, $\mathrm{pH} 7.0) / 10 \%$ acetonitrile $(\mathrm{v} / \mathrm{v})$. Flow rate: 1 $\mathrm{mL} / \mathrm{min}$. $\lambda$ : $215 \mathrm{~nm}$. Temperature: $35^{\circ} \mathrm{C}$.
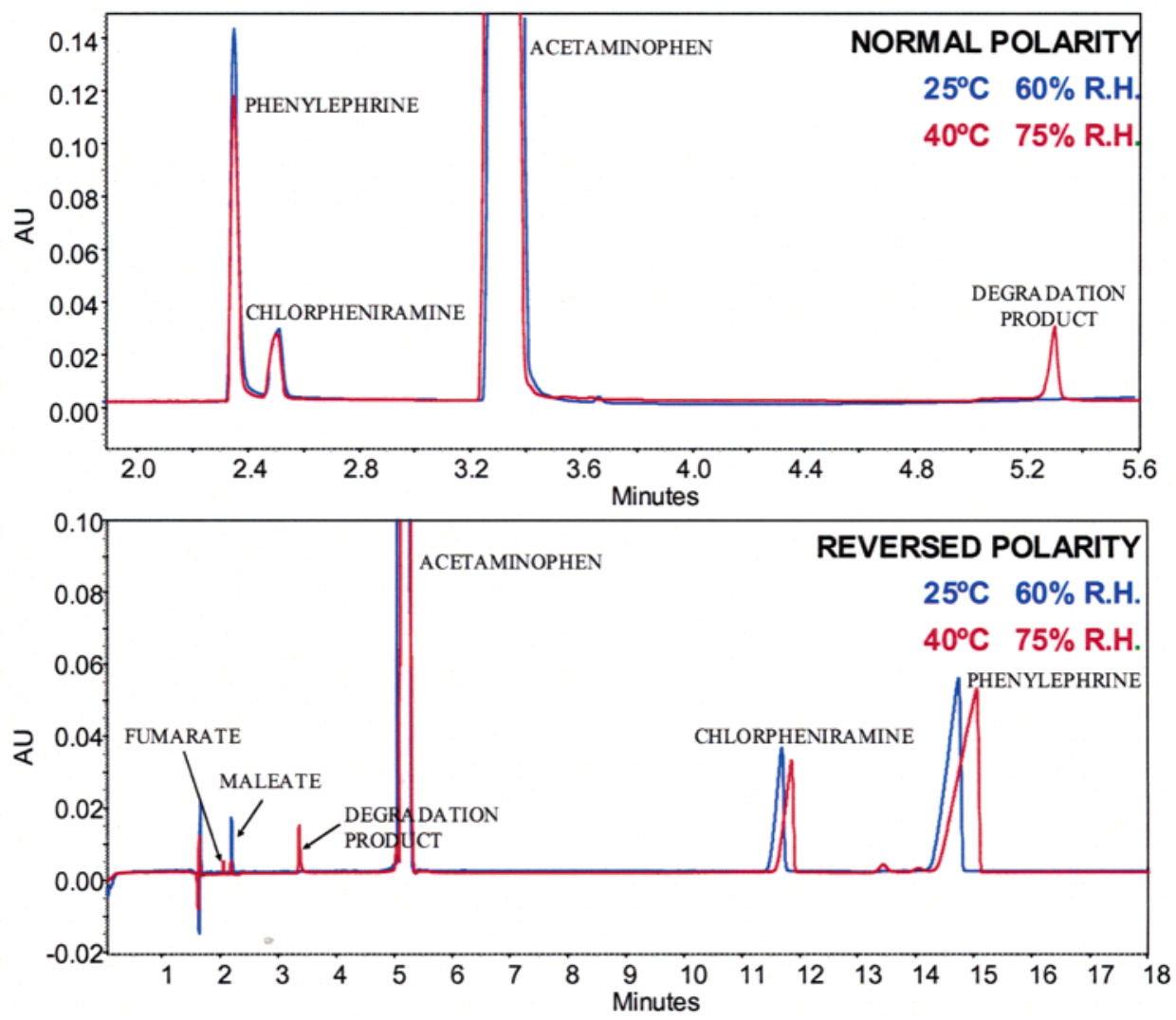

Figure 2. Electropherograms of capsules stored in climatic chambers at different temperatures and RH after 6 months obtained using normal and reversed CE methods. See conditions in the text.

inverse probe. ${ }^{1} \mathrm{H}$ and ${ }^{13} \mathrm{C}$ spectra were acquired using $64 \mathrm{~K}$ and $32 \mathrm{~K}$ data points, respectively. The proton chemical shifts were referenced to the residual water signal at $4.77 \mathrm{ppm}\left(25^{\circ} \mathrm{C}\right)$ and the carbon chemical shifts to internal dioxane at $67.2 \mathrm{ppm}$. Absolute value COSY (correlation spectroscopy), phase-sensitive HSQC (heteronuclear single-quantum correlation), and HMBC (heteronuclear multiple-bond correlation) experiments were acquired using gradient selection techniques. Acquisition data matrices were defined by $1 \mathrm{~K} \times 128$ points in $t_{2}$ and $t_{1}$, respectively.
Data were processed using the XWINNMR Bruker program on a Silicon Graphics computer.

\section{RESULTS AND DISCUSSION}

HPLC. An unidentified degradation product with a UV spectrum similar to that observed for phenylephrine appeared after 6 months during the stability assay of the capsules described above. It showed increasing concentrations at increasing storage temperature as can be observed in Figure 1. Simultaneously, a 


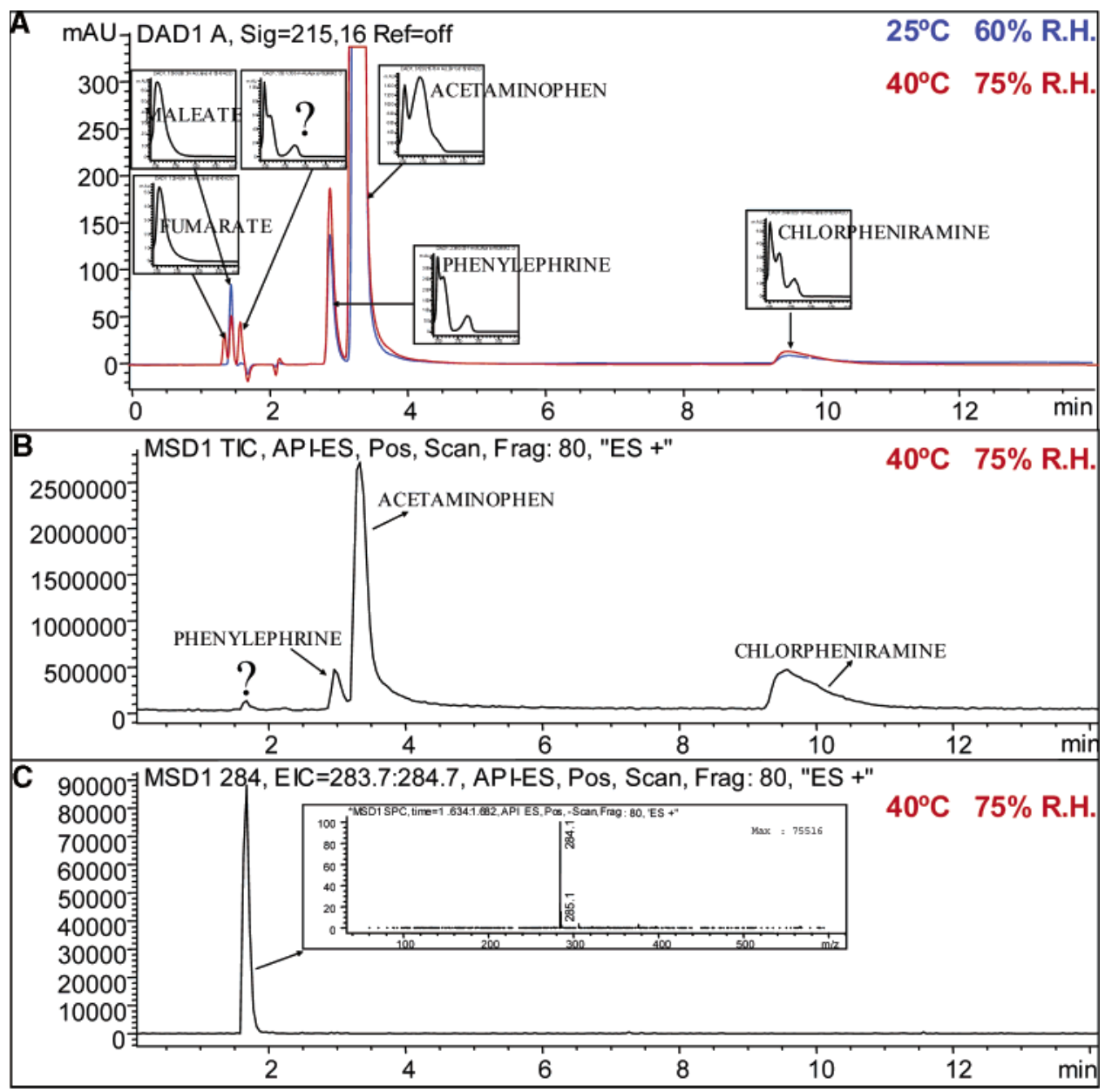

Figure 3. (A) DAD chromatograms of capsules after 6 months storage in climatic chambers at different temperatures and RH with the LC/MS method. (B) TIC of capsules stored at $40^{\circ} \mathrm{C}$ and $75 \% \mathrm{RH}$. (C) EIC of the degradation product with its LC/ESI/MS spectrum. Column: Discovery HS PEG $(5 \mu \mathrm{m}, 4.6 \times 150 \mathrm{~mm})$. Mobile phase: $90 \%(10 \mathrm{mM}$ ammonium acetate, $\mathrm{pH} 7.0) / 10 \%$ acetonitrile $(\mathrm{v} / \mathrm{v})$. Flow rate: $1 \mathrm{~mL} / \mathrm{min} . \lambda: 215$ nm. Temperature: $35^{\circ} \mathrm{C}$.

decrease in the peak of phenylephrine as well as in the peak of maleate was detected in the capsules stored into climatic chambers at $40{ }^{\circ} \mathrm{C}$ and $75 \% \mathrm{RH}$. The decrease in the phenylephrine peak reached $20 \%$ of the active compound after 6 months in some formulations.

CE. To obtain complete information of the degradation profile, two $\mathrm{CE}$ methods were developed, one using normal and the other reversed polarity. At first instance, the $\mathrm{pH}$ was selected by running the three standards at $\mathrm{pH} 3,7$, and 12 , adding to the buffer 0.05 $\mathrm{mM}$ SDS for normal polarity and $0.5 \mathrm{mM} \mathrm{CTAB}$ for reversed polarity, applying a constant current of $100 \mu \mathrm{A}(\sim 30 \mathrm{kV})$. In both cases, the best results were found at $\mathrm{pH} 7$. Therefore, buffers with $\mathrm{pH}$ ranging from 6.00 to 8.00 with $0.2 \mathrm{pH}$ increments were tested as well as the effect of buffer concentration in the background electrolyte (BGE), the concentration of surfactants, SDS and CTAB (always under the critical micellar concentration), and the presence of a small percentage of organic modifier in the BGE, methanol, or acetonitrile.

We succeeded in separating the three analytes (acetaminophen, phenylephrine, chlorpheniramine), plus 4-nitrophenol, and the excipients within a run time shorter than $6 \mathrm{~min}$, using a buffer composed of $40 \mathrm{mM}$ phosphate buffer with $0.5 \mathrm{mM}$ SDS at $\mathrm{pH} 6.20$ and normal polarity. This method was validated and

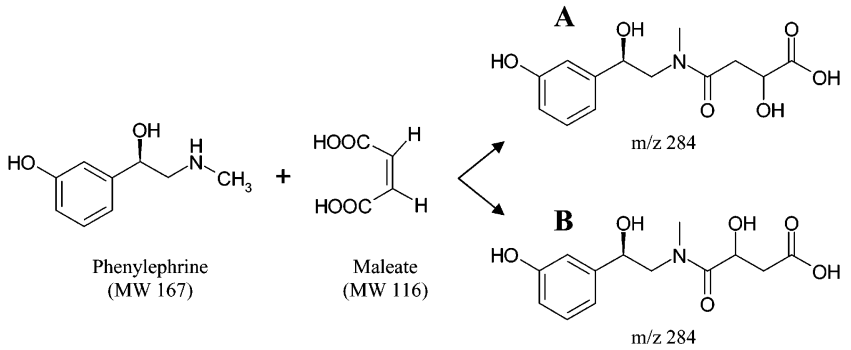

Figure 4. Possible structures of the degradation product formed in capsules stored at $40^{\circ} \mathrm{C}$ and $75 \% \mathrm{RH}$ after 6 months.

compared with HPLC for the dissolution test of the capsules. ${ }^{3}$ Under these conditions, acetaminophen, which is the biggest peak, coelutes with the other neutral impurities.

Using the method with CTAB and reversed polarity,, the three analytes, including the co-ion maleate and the excipients, were separated in a 18-min run, employing the same current and a buffer composed of $40 \mathrm{mM}$ phosphate buffer with $0.5 \mathrm{mM} \mathrm{CTAB}$ at $\mathrm{pH}$ 6.40. In both cases, the UV detection was performed at 200 $\mathrm{nm}$ and the capillary temperature was maintained at $25^{\circ} \mathrm{C}$.

When these two methods were applied to the stability study of capsules, it was found that a new peak with a spectrum similar 


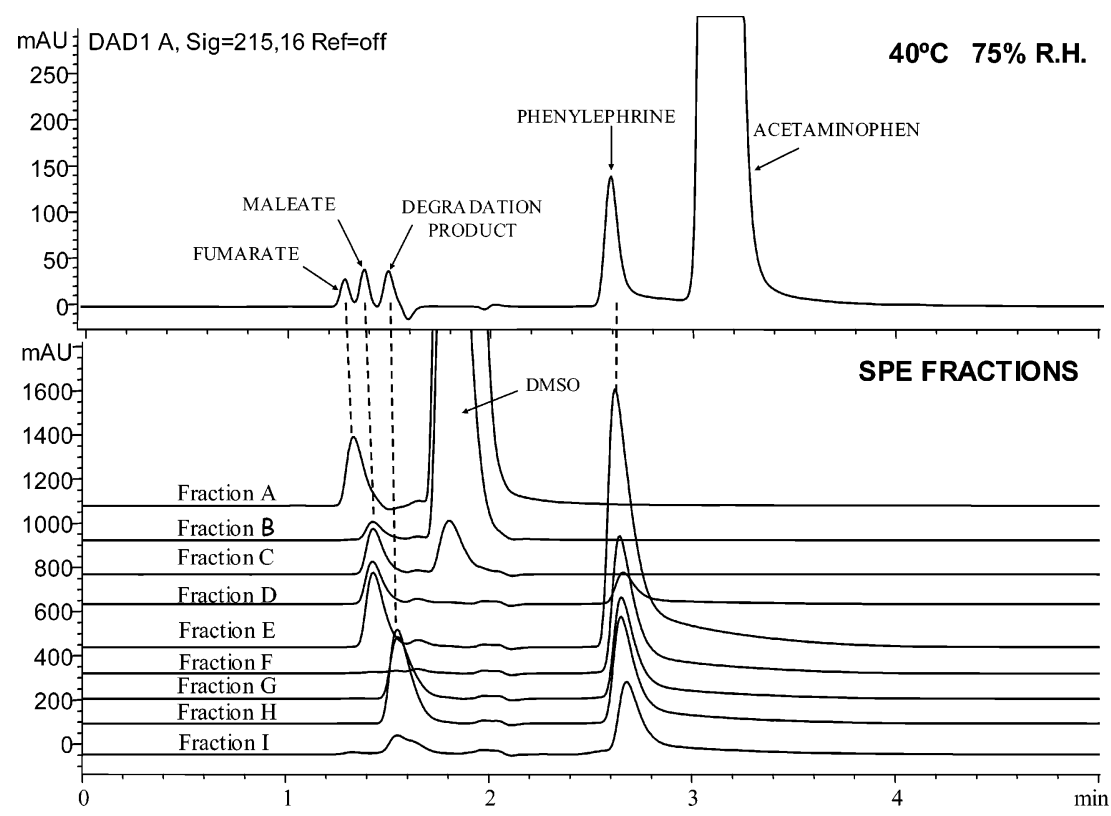

Figure 5. Chromatograms of capsules stored in climatic chambers at $40^{\circ} \mathrm{C}$ and $75 \% \mathrm{RH}$ and the fractions of the SPE with the LC/MS method on a Discovery HS PEG column $(5 \mu \mathrm{m}, 4.6 \times 150 \mathrm{~mm})$.

to that observed for phenylephrine appeared in samples stored at $40{ }^{\circ} \mathrm{C}$ and $75 \% \mathrm{RH}$ (Figure 2). In the electropherograms with normal polarity (Figure 2A), a decrease in the peak of phenylephrine was detected, whereas with reversed polarity (Figure 2B), a decrease in the maleate peak and a new peak with a UV spectrum similar to that found for maleate were also detected. This last compound was identified as fumarate, the trans isomer of the maleate (co-ion of chorpheniramine). This identification was carried out by both comparing the migration times and spiking with the pure standard.

Moreover, the $\mathrm{CE}$ methods provide additional information about the nature of the impurity. Thus, it was concluded that the impurity must be an anion at the working $\mathrm{pH}$, possibly an acidic compound, of large charge/mass ratio as can be deduced from its migration times in normal and reversed polarity.

LC/MS. The LC/MS method previously developed to analyze the degraded samples under heating, acid, base, UV radiation, and oxidation stress conditions ${ }^{6}$ was used to obtain the molecular weight of the degradation product formed in the samples stored at $40{ }^{\circ} \mathrm{C}$ and $75 \% \mathrm{RH}$ after 6 months. In the chromatograms of Figure $3 \mathrm{~A}$, it can be observed that when phenylephrine and maleate decreased, two new peaks appeared. One of them corresponded to the impurity with the same UV spectrum as phenylephrine, and the second one, with the same UV spectrum as maleate was the identified fumarate. The impurity showed a $m / z$ of 284 in the positive mode (Figure 3B and C) that could correspond with an ion formed from the sum of phenylephrine (MW 167) and maleate (MW 116). At this point and summarizing the information that provided the HPLC, CE, and LC/MS, we proposed two possible structures that are shown in Figure 4. To obtain detailed structural information on this degradation product, its isolation was required.

SPE Isolation. The degradation product was a minor component in the sample $(\sim 0.20 \%$ in total weight), and therefore, its purification required two steps. First, SPE was used to obtain a

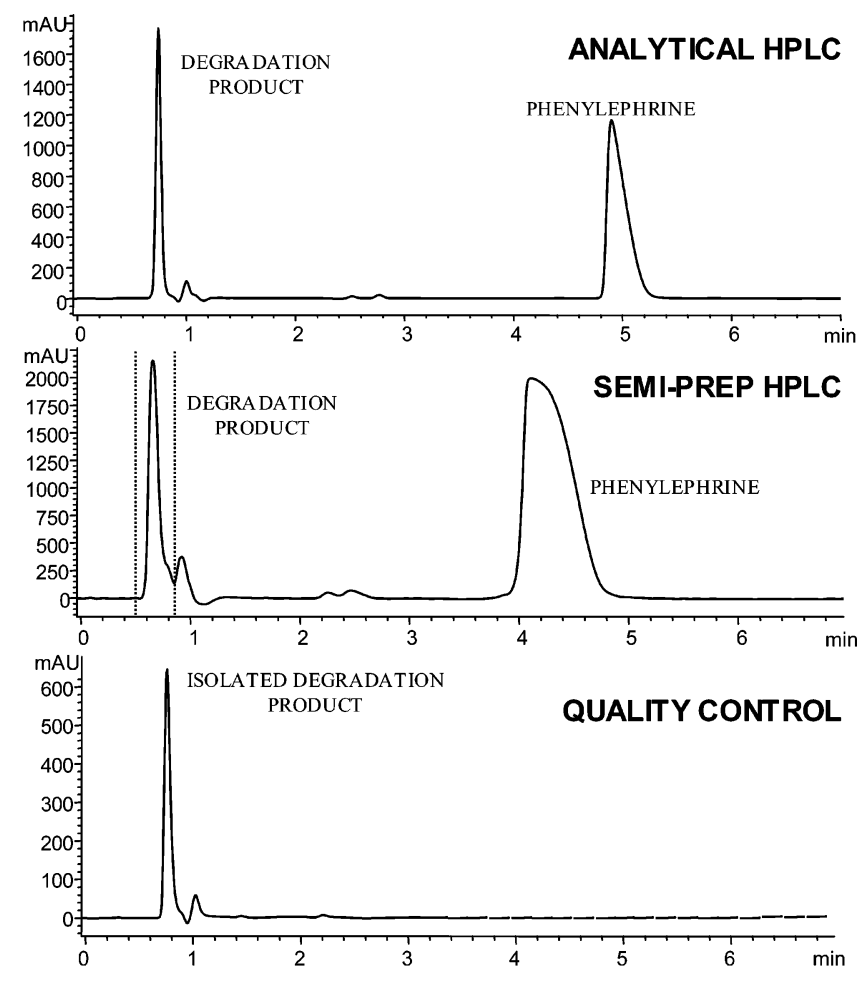

Figure 6. (a) Analytical chromatogram of the SPE fraction containing the degradation product and phenylephrine on a Discovery HS F5 $100 \times 4.6 \mathrm{~mm}, 5 \mu \mathrm{m}$, kept at $35{ }^{\circ} \mathrm{C}$ under isocratic conditions with $40 \%$ (10 mM ammonium acetate, at $\mathrm{pH} 7) / 60 \%$ acetonitrile at 1 $\mathrm{mL} / \mathrm{min}$. (b) Semiprep chromatogram on a Discovery HS F5 $100 \times$ $10 \mathrm{~mm}, 5 \mu \mathrm{m}$, kept at $35^{\circ} \mathrm{C}$ at $5 \mathrm{~mL} / \mathrm{min}$. (c) Chromatogram of the isolated degradation product.

rich fraction in the target degradation product, and second, HPLC was used to get a fine isolation of this solute.

Oasis HLB cartridges for SPE were used. This sorbent is a macroporous copolymer made of two compounds, a lipophilic 

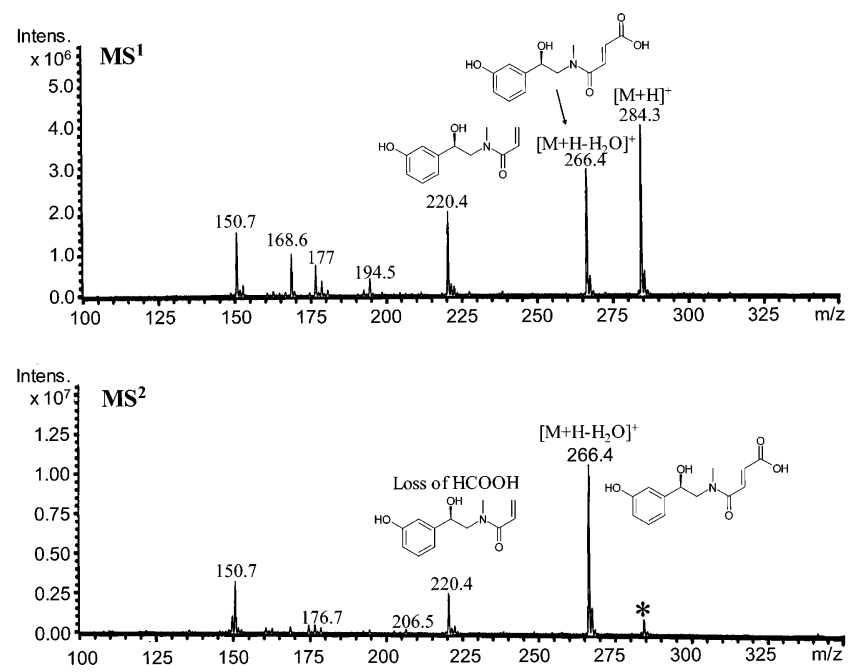

Figure 7. $M S$ and $M S / M S$ spectra of the isolated degradation product.

monomer, divinylbenzene, and a hydrophilic monomer, $\mathrm{N}$-vinylpyrrolidone. The lipophilic monomer will provide a reversephase-type mechanism for trapping analytes, while the hydrophilic monomer will give wetting properties to the sorbent and better extraction of polar compounds. The polymer itself also interacts with the analytes strongly, by both hydrogen bonding and hydrophobic interaction, enabling its application for various analytes.

The isolation was a difficult task because the polarity of the degradation product was very close to the polarity of maleate and phenylephrine, its spectrum was very similar to phenylephrine, but they presented a great imbalance in concentrations. The mobile phase used was $10 \mathrm{mM}$ ammonium acetate at $\mathrm{pH} 7$ and methanol. During the elution process, no more than 5\% methanol was used because the target compound was very polar. Collected fractions were concentrated and analyzed by HPLC. Results are shown in Figure 5. The degradation product was found in fractions $\mathrm{G}$ and $\mathrm{H}$ together with phenylephrine. Therefore, those fractions had to be purified in a second step by HPLC in order to obtain a highly pure compound (>95\%).

HPLC Isolation. Separation with conventional C18 columns, between the unidentified degradation product and phenylephrine, was not enough to collect a pure fraction for structural identification. We have observed, and it has been previously described, ${ }^{16-18}$ that generally, bases are longer retained on the HS F5 (pentafluorophenylpropyl phase) than on a $\mathrm{C} 18$ alkyl phase. Moreover, aromatic polar compounds that elute too closely to the void volume on $\mathrm{C} 18$ columns are sufficiently retained by Discovery HS F5, and therefore, a HS F5 $100 \times 4.6 \mathrm{~mm}, 5 \mu \mathrm{m}$ was selected. On the other hand, the Discovery HS F5 exhibits both reversed-phase and normal-phase characteristics for certain basic analytes. In the normal phase, many basic compounds are highly retained using mobile phases in excess of organic modifier concentration. During the optimization of the mobile phase, these reversed- and normalphase behaviors were observed under certain mobile-phase conditions for phenylephrine. At low organic proportion, retention decreases with increasing percent organic following reversedphase behavior. Interestingly, at higher percent of organic solvent, retention increases with increasing percent of organic, following normal-phase behavior, which can be especially advantageous for $\mathrm{LC} / \mathrm{MS}$. At this point, there is not a clear consensus regarding the retention mechanisms to explain such behavior. For the purification, we choose the mobile-phase composition that gave enough retention for phenylephrine with a high percent of acetonitrile and short run time. Thus, a $60 \%$ acetonitrile could be

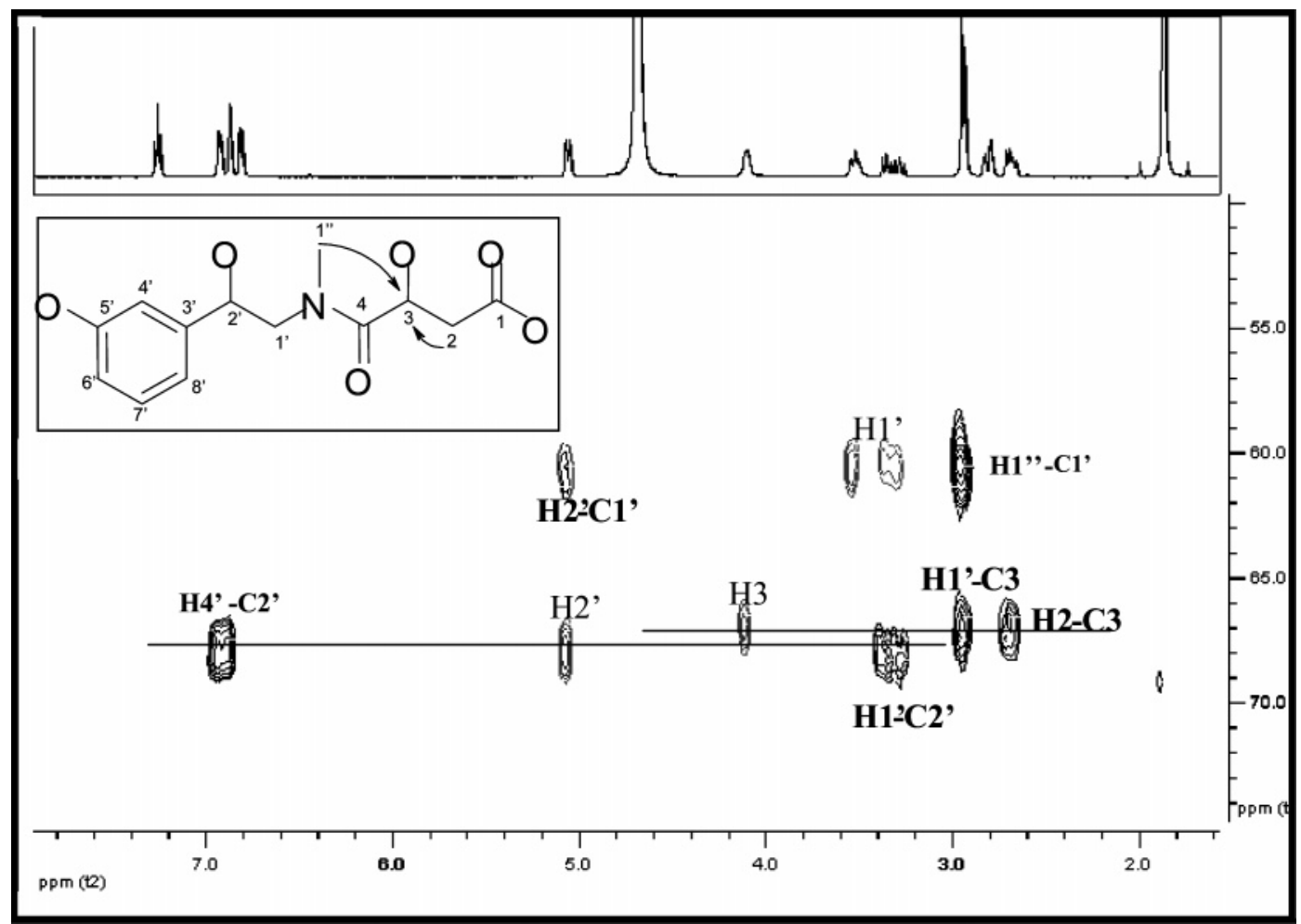

Figure 8. Expansion of the HSQC (in gray) and HMBC (in black). The correct isomer with the key long-range ${ }^{1} \mathrm{H}-{ }^{13} \mathrm{C}$ correlation is superimposed on the spectrum. 
Table 1. Chemical Shifts of Protons Referenced to the Residual Water Signal at 4.77 ppm $\left(25^{\circ} \mathrm{C}\right)$ and Chemical Shifts of Carbons Referenced to Internal Dioxane at $67.2 \mathrm{ppm}$ at $50{ }^{\circ} \mathrm{C}$

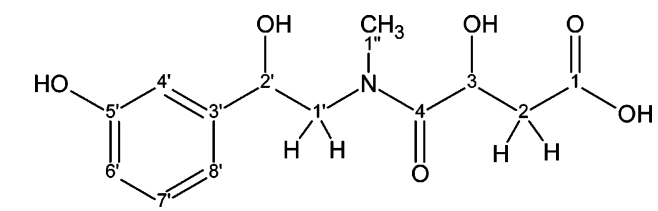

$\begin{array}{lcc}\text { atom } & \begin{array}{c}{ }^{1} \mathrm{H} \text { chemical } \\ \text { shift (ppm) }\end{array} & \begin{array}{c}{ }^{13} \mathrm{C} \text { chemica } \\ \text { shift (ppm) }\end{array} \\ 1 & & 186.1 \\ 2 \mathrm{a} & 2.77 & 36.9 \\ 2 \mathrm{~b} & 2.89 & \\ 3 & 4.18 & 70.5 \\ 4 & & 174.8 \\ 1^{\prime} \mathrm{a} & 3.40 & 63.2 \\ 1^{\prime} \mathrm{b} & 3.62 & 70.6 \\ 2^{\prime} & 5.14 & 144.4 \\ 3^{\prime} & & 115.9 \\ 4^{\prime} & 6.95 & 158.9 \\ 5^{\prime} & & \end{array}$

employed versus $10 \%$ in the standard method, and sample concentration was easier.

The optimized analytical method (chromatogram a, Figure 6) was directly transferred to semipreparative HPLC (chromatogram b) employing a Discovery HS F5 $100 \times 10 \mathrm{~mm}, 5 \mu \mathrm{m}$ kept at 35 ${ }^{\circ} \mathrm{C}$ under isocratic conditions with $40 \% 10 \mathrm{mM}$ ammonium acetate at pH 7 and $60 \%$ acetonitrile at $5 \mathrm{~mL} / \mathrm{min}$. Finally, it was possible to isolate $7 \mathrm{mg}$ of the degradation product from $4.5 \mathrm{~g}$ of sample. The chromatogram of the isolated compound (chromatogram c) showed a purity higher than $95 \%$. Purity was also confirmed with the UV HPLC method and the LC/MS method on a PEG column (data not shown).

MS/MS. To confirm the structure of the degradation product, MS/MS experiments were performed using an ion trap system, which offers the potential for much greater and more selective fragmentation, and therefore information, than a single quadrupole instrument. The purified compound was introduced by infusion and analyzed by APCI and ESI in the positive mode with $m / z$ scan from 100 to 350. Data for APCI are shown in Figure 7. The compound gave a molecular ion at $m / z 284[\mathrm{M}+\mathrm{H}]$ in good agreement with the result from $\mathrm{LC} / \mathrm{MS}$. It generated a fragment ion at $m / z 266$, which can be assigned as the dehydrated product.

(16) Needham, S. R.; Jeanville, P. M.; Brown, P. R.; Estape, E. S J. Chromatogr., $B$ 2000, 748, 77-87.

(17) Needham, S. R.; Brown, P. R. J. Pharm. Biomed. Anal. 2000, 23, 597-605.

(18) Lee, C. H.; Chang, T. C.; Lee, S. L.; Den, T. G. Fresenius' Z. Anal. Chem. 1987, 328, 37-40.
The $m / z 220$ can be the loss of formic acid (-46) from the dehydrated product. The $m / z 168$ can probably correspond to phenylephrine, which demonstrates that the degradation product is related to this compound, and the $m / z 151$ is surely the phenylephrine dehydrated. When the parent ion $m / z 284$ was selected and fragmentized to MS/MS experiment, the same fragments with $m / z 220$ and 151 were detected.

NMR. The degradation product was fully characterized through the combination of ${ }^{1} \mathrm{H},{ }^{13} \mathrm{C}, \mathrm{COSY}, \mathrm{HSQC}$, and $\mathrm{HMBC}$ experiments. Inspection of the ${ }^{1} \mathrm{H}$ spectrum revealed the presence of four aromatic and seven aliphatic protons. The exchangeable protons were not detected, as expected for a sample in $\mathrm{D}_{2} \mathrm{O}$. Assignment of the resonances was achieved through the ${ }^{1} \mathrm{H}-{ }^{1} \mathrm{H}$ correlations observed in a COSY spectrum and the one-bond ${ }^{1} \mathrm{H}-$ ${ }^{13} \mathrm{C}$ correlations observed in the HSQC spectrum. The correct isomer (A or $\mathrm{B}$ ) was determined through a $\mathrm{HMBC}$ spectrum: the methyl protons showed a long-range ${ }^{1} \mathrm{H}-{ }^{13} \mathrm{C}$ correlation to the methine carbon $\left(\mathrm{C}_{3}\right)$, whereas correlation to the methylene carbon was not present. The observed correlation is only consistent with isomer B (Figure 8) as five-bond correlations are unlikely to be detected in a HMBC. The proton and carbon chemical shifts are collected in Table 1 . The corresponding compound is named 3-hydroxy- $N$-[2-hydroxy-2-(3-hydroxyphenyl) ethyl]- $N$-methylsuccinamic acid.

\section{CONCLUSIONS}

A new compound has been isolated and identified for the first time in pharmaceutical formulations against the common cold containing acetaminophen, phenylephrine, and chlorpheniramine maleate. For isolation, SPE and HPLC methods have been developed and applied. For structural elucidation CE, LC/MS, MS/MS, ${ }^{1} \mathrm{H}$ NMR, ${ }^{13} \mathrm{C}$ NMR, COSY, HSQC, and HMBC have been employed. The identification of this degradation product has permitted us to understand and to confirm its formation mechanism. There is evidence that this compound is formed from the interaction between phenylephrine and maleate. It is very important to remark that it has not been described in the literature and that it has been identified in several commercial preparations containing phenylephrine hydrochloride and chlorpheniramine maleate. Further toxicological studies will be needed to assess its toxicity.

\section{ACKNOWLEDGMENT}

The authors thank Lilly laboratories for the technical support, especially to A. Rivera and the NMR group, and Cinfa laboratories for providing the test materials.

Received for review June 28, 2004. Accepted October 18, 2004.

AC0490550 